Open J. Math. Sci., Vol. 2(2018), No. 1, pp. 240 - 252

Website: https://pisrt.org/psr-press/journals/oms/

ISSN: 2523-0212 (Online) 2616-4906 (Print)

http://dx.doi.org/10.30538/oms2018.0032

\title{
OSCILLATORY BEHAVIOR OF SECOND ORDER NONLINEAR DIFFERENCE EQUATIONS WITH A NON-POSITIVE NEUTRAL TERM
}

\author{
SAID R. GRACE, SHURONG SUN ${ }^{1}$, LIMEI FENG, YING SUI
}

\begin{abstract}
We shall present new oscillation criteria of second order nonlinear difference equations with a non-positive neutral term of the for $\Delta\left(a(t)(\Delta(x(t)-p(t) x(t-k)))^{\gamma}\right)+q(t) x^{\beta}(t+1-m)=0$, with positive coefficients. Examples are given to illustrate the main results.

Mathematics Subject Classification: Write MSC.

Key words and phrases: Oscillation; Second order; Neutral difference equa-
\end{abstract} tion; Non-positive neutral term.

\section{Introduction}

This paper deals with oscillatory behavior of all solutions of the nonlinear second order difference equations with a non-positive neutral term of the form

$$
\Delta\left(a(t)(\Delta(x(t)-p(t) x(t-k)))^{\gamma}\right)+q(t) x^{\beta}(t+1-m)=0 .
$$

We assume that

(i) $\gamma, \beta$ are the ratios of positive odd integers;

(ii) $\{a(t)\},\{p(t)\}$ and $\{q(t)\}$ are positive real sequences for $t \geqslant t_{0}$, and $0<$ $p(t)<p_{0}<1$;

(iii) $k$ is a positive integer and $m$ is a nonnegative integer;

(iv) $h(t)=t-m+k+1 \leqslant t$, that is $m \geqslant k+1$.

We let

$$
A(v, u)=\sum_{s=u}^{v-1} \frac{1}{a^{1 / \gamma}(s)}, v \geqslant u \geqslant t_{0},
$$

Received 28 March 2018. Revised 8 September 2018

1 Corresponding Author

(C) 2018 Said R. Grace, Shurong Sun, Limei Feng, Ying Sui. This is an open access article distributed under the Creative Commons Attribution License, which permits unrestricted use, distribution, and reproduction in any medium, provided the original work is properly cited. 
and assume that

$$
A\left(t, t_{0}\right) \rightarrow \infty \text { as } t \rightarrow \infty
$$

Let $\theta=\max \{k, m-1\}$. By a solution of equation (1), we mean a real sequence $\{x(t)\}$ defined for all $t \geqslant t_{0}-\theta$ and satisfies equation (1) for all $t \geqslant t_{0}$. A solution of equation (1) is called oscillatory if its terms are neither eventually positive nor eventually negative, otherwise it is called non-oscillatory. If all solutions of the equation are oscillatory then the equation itself called oscillatory.

In recent years, there has been much research activity concerning the oscillation and asymptotic behavior of solutions of various classes of difference equations see $[1,2,3,4,5,6,7,8,9,10,11]$ and the references cited therein. Meanwhile, there also have been numerous research for second order neutral functional difference equations, due to the comprehensive use in natural science and theoretical study. Some interesting recent results on the oscillatory and asymptotic behavior of second order difference equations can be found in $[12,13,14,15,16,17,18$, $19,20,21,22]$. However, it seems that there are no known results regarding the oscillation of second order difference equations of type (1). More exactly existing literature does not provide any criteria which ensure oscillation of all solutions of equation (1). In view of the above motivation, our aim in this paper is to present sufficient conditions which ensure that all solutions of (1) are oscillatory.

\section{Main results}

For $t \geqslant T$ for some $T \geqslant t_{0}$ we let

$$
\mu(t)=a^{1 / \gamma}(t) A(t, T) \text { and } Q(t)=\sum_{s=t}^{\infty} q(s) .
$$

We begin with the following new result.

Theorem 2.1. Let conditions $(i)$ - (iv) and equation (2) hold. If there exists a positive non-decreasing sequence $\{\rho(t)\}$ such that

$$
\begin{aligned}
& \limsup _{t \rightarrow \infty}\left(\rho(t) Q(t)+\sum_{s=t_{2}}^{t}\left[\rho(s) q(s)-\frac{\gamma^{\gamma}}{(1+\gamma)^{1+\gamma}} \frac{a(t-m+1)}{(\beta g(s))^{\gamma}}\left(\frac{(\Delta \rho(s))^{\gamma+1}}{\rho^{\gamma}(s)}\right)\right]\right) \\
& =\infty
\end{aligned}
$$

where

$$
\begin{gathered}
g(t)= \begin{cases}1, & \text { when } \beta=\gamma, \\
c\left(A^{(\gamma-\beta) / \beta}(t)\right), & \text { when } \beta>\gamma \text { for some constant } c>0,\end{cases} \\
\quad \limsup _{t \rightarrow \infty} \sum_{s=h(t)}^{t-1} A^{\beta}(h(t), h(s)) q(s)>1, \text { when } \beta=\gamma
\end{gathered}
$$

and

$$
\limsup _{t \rightarrow \infty} \sum_{s=h(t)}^{t-1} A^{\beta}(h(t), h(s)) q(s)>c>0, \text { when } \beta>\gamma,
$$

then equation (1) is oscillatory. 
Proof. Let $x(t)$ be a non-oscillatory solution of equation (1), say $x(t)>0, x(t-$ $m+1)>0, x(t-k)>0$ for $t \geqslant t_{1}$ for some $t_{1} \geqslant t_{0}$. It follows from equation (1) that

$$
\Delta\left(a(t)(\Delta y(t))^{\gamma}\right)=-q(t) x^{\beta}(t-m+1),
$$

where $y(t)=x(t)-p(t) x(t-k)$. Hence $a(t)(\Delta y(t))^{\gamma}$ is decreasing and of one sign. That is, there exists a $t_{2} \geqslant t_{1}$ such that $\Delta y(t)>0$ or $\Delta y(t)<0$ for $t \geqslant t_{2}$. We claim that $\Delta y>0$ for $t \geqslant t_{2}$. To prove it, we assume that $\Delta y(t)<0$ for $t \geqslant t_{2}$. Then

$$
a(t)(\Delta y(t))^{\gamma} \leqslant-c \text { for } t \geqslant t_{2},
$$

where $c=-a\left(t_{2}\right)\left(\Delta y\left(t_{2}\right)\right)^{\gamma}>0$. Thus, we conclude that

$$
y(t) \leqslant y\left(t_{2}\right)-c^{1 / \gamma} \sum_{s=t_{2}}^{t-1} a^{-1 / \gamma}(s) .
$$

By virtue of equation (2), $\lim _{t \rightarrow \infty} y(t)=-\infty$. Now, we consider the following two cases:

Case 1. If $x(t)$ is unbounded, then there exists a sequence $\left\{t_{n}\right\}$ such that $\lim _{n \rightarrow \infty} t_{n}=\infty$ where $x\left(t_{n}\right)=\max \left\{x(s): t_{0} \leqslant s \leqslant t_{n}\right\}$. Since $t_{n}-k>t_{0}$ for all sufficiently large $n$,

$$
x\left(t_{n}-k\right)=\max \left\{x(s): t_{0} \leqslant s \leqslant t_{n}-k\right\} \leqslant \max \left\{x(s): t_{0} \leqslant s \leqslant t_{n}\right\}=x\left(t_{n}\right) .
$$

Therefore, for all large $n$,

$$
y\left(t_{n}\right)=x\left(t_{n}\right)-p\left(t_{n}\right) x\left(\tau\left(t_{n}\right)\right) \geqslant\left(1-p\left(t_{n}\right)\right) x\left(t_{n}\right)>0,
$$

where $\tau(t)=t-k$, which contradicts the fact that $\lim _{t \rightarrow \infty} y(t)=-\infty$.

Case 2. If $x(t)$ is bounded, then $y(t)$ is also bounded, which contradicts $\lim _{t \rightarrow \infty} y(t)=$ $-\infty$. This completes the prove of the claim and conclude that $\Delta y(t)>0$ for $t \geqslant t_{2}$.

Next, we have two cases to consider:

(I) $y(t)>0$; (II) $y(t)<0$, for $t \geqslant t_{2}$.

First assume that (I) holds. In view of equation (7) and $x(t) \geqslant y(t)$, we have

$$
\Delta\left(a(t)(\Delta y(t))^{\gamma}\right) \leqslant-q(t) y^{\beta}(t-m+1) \leqslant 0 .
$$

It follows that

$$
\begin{aligned}
y(t) & =y\left(t_{2}\right)+\sum_{s=t_{2}}^{t-1} \frac{\left(a(s)(\Delta y(s))^{\gamma}\right)^{1 / \gamma}}{a^{1 / \gamma}(s)} \\
& \geqslant a^{1 / \gamma}(t)(\Delta y(t)) \sum_{s=t_{2}}^{t-1} a^{-1 / \gamma}(s) \\
& :=\mu(t) \Delta y(t) .
\end{aligned}
$$


Summing equation (8) from $t$ to $u$, letting $u \rightarrow \infty$ and using the fact that $y(t)$ is increasing, we have

$$
\begin{aligned}
a(t)(\Delta y(t))^{\gamma} & \geqslant \sum_{s=t}^{\infty} q(s) y^{\beta}(s-m+1) \\
& \geqslant y^{\beta}(t-m+1)\left(\sum_{s=t}^{\infty} q(s)\right) \\
& :=Q(t) y^{\beta}(t-m+1) .
\end{aligned}
$$

Suppose that $y(t)>0$ for $t \geqslant t_{2}$. Define

$$
w(t)=\rho(t) \frac{a(t)(\Delta y(t))^{\gamma}}{y^{\beta}(t-m)} \text { for } t \geqslant t_{2} .
$$

Then, it follows that

$$
w(t)=\rho(t) \frac{a(t)(\Delta y(t))^{\gamma}}{y^{\beta}(t-m)} \geqslant \rho(t)\left(\sum_{s=t}^{\infty} q(s)\right) .
$$

Now,

$$
\begin{aligned}
\Delta w(t) & =\Delta\left(\frac{\rho(t)}{y^{\beta}(t-m)}\right)\left(a(t+1)(\Delta y(t+1))^{\gamma}\right)+\Delta\left(a(t)(\Delta y(t))^{\gamma}\right)\left(\frac{\rho(t)}{y^{\beta}(t-m)}\right) \\
& \leqslant-\rho(t) q(t)+\left(\frac{\Delta \rho(t)}{\rho(t+1)}\right) w(t+1)-\left(\frac{\rho(t)}{\rho(t+1)}\right) \frac{\Delta y^{\beta}(t-m)}{y^{\beta}(t-m)} w(t+1) .
\end{aligned}
$$

By the corollary of the Keller chain rule, for $0<\beta \leq 1$, we have

$$
\begin{aligned}
\Delta y^{\beta}(t-m) & =\beta \int_{0}^{1}\left[h y(g(t-m+1)+(1-h) y(t-m)]^{\beta-1} \Delta y(t-m) d h\right. \\
& \geq \beta \int_{0}^{1}[h y(t-m+1)+(1-h) y(t-m+1)]^{\beta-1} \Delta y(t-m) d h \\
& =\beta y^{\beta-1}(t-m+1) \Delta y(t-m), \quad 0<\beta \leq 1,
\end{aligned}
$$

then using this in (13), we get

$$
\begin{aligned}
\Delta w(t) & \leqslant-\rho(t) q(t)+\left(\frac{\Delta \rho(t)}{\rho(t+1)}\right) w(t+1) \\
& -\beta\left(\frac{\rho(t)}{\rho(t+1)}\right) \frac{y^{\beta-1}(t-m+1) \Delta y(t-m)}{y^{\beta}(t-m)} w(t+1) \\
& \leqslant-\rho(t) q(t)+\left(\frac{\Delta \rho(t)}{\rho(t+1)}\right) w(t+1)-\beta\left(\frac{\rho(t)}{\rho(t+1)}\right) \frac{\Delta y(t-m)}{y(t-m)} w(t+1) .
\end{aligned}
$$


And for $\beta>1$, we have

$$
\begin{aligned}
\Delta y^{\beta}(t-m) & =\beta \int_{0}^{1}\left[h y(g(t-m+1)+(1-h) y(t-m)]^{\beta-1} \Delta y(t-m) d h\right. \\
& \geq \beta \int_{0}^{1}[h y(t-m)+(1-h) y(t-m)]^{\beta-1} \Delta y(t-m) d h \\
& =\beta y^{\beta-1}(t-m) \Delta y(t-m), \quad \beta>1,
\end{aligned}
$$

then using this in equation (13), we get

$$
\Delta w(t) \leqslant-\rho(t) q(t)+\left(\frac{\Delta \rho(t)}{\rho(t+1)}\right) w(t+1)-\beta\left(\frac{\rho(t)}{\rho(t+1)}\right) \frac{\Delta y(t-m)}{y(t-m)} w(t+1) .
$$

Thus, by equation (14) and equation (15), we obtain equation (15) hold for all $\beta>0$. Since $\left(a(t)(\Delta y(t))^{\gamma}\right)$ is decreasing, we have

$$
\frac{\Delta y(t-m)}{\Delta y(t)} \geqslant\left(\frac{a(t)}{a(t-m)}\right)^{1 / \gamma} \text { and } \frac{w(t+1)}{\rho(t+1)} \leqslant \frac{w(t)}{\rho(t)} .
$$

Using equation (16) in equation (15), we obtain

$\Delta w(t) \leqslant-\rho(t) q(t)+\left(\frac{\Delta \rho(t)}{\rho(t+1)}\right) w(t+1)-\beta\left(\frac{\rho(t)}{\rho(t+1)}\right)\left(\frac{a(t)}{a(t-m)}\right)^{1 / \gamma}\left(\frac{\Delta y(t)}{y(t-m)}\right) w(t+$ $1)$.

Now,

$$
\frac{\Delta y(t)}{y^{\gamma / \beta}(t-m)}=\rho^{-1 / \gamma}(t) a^{-1 / \gamma}(t) w^{1 / \gamma}(t) \geqslant \rho^{-1 / \gamma}(t) a^{-1 / \gamma}(t)\left(\frac{\rho(t)}{\rho(t+1)}\right)^{1 / \gamma} w^{1 / \gamma}(t+1) .
$$

Thus,

$\Delta w(t) \leqslant-\rho(t) q(t)+\left(\frac{\Delta \rho(t)}{\rho(t+1)}\right) w(t+1)-\frac{\beta}{a^{1 / \gamma}(t-m)}\left(\frac{\rho(t)}{\rho^{1+1 / \gamma}(t+1)}\right) w^{1+(1 / \gamma)}(t+$ 1) $y^{(\gamma-\beta) / \beta}(t-m)$

and so,

$$
\begin{aligned}
\Delta w(t) \leqslant & -\rho(t) q(t)+\left(\frac{\Delta \rho(t)}{\rho(t+1)}\right) w(t+1) \\
& -\frac{\beta \rho(t)}{a^{1 / \gamma}(t-m) \rho^{1+1 / \gamma}(t+1)} w^{1+1 / \gamma}(t+1) y^{(\gamma-\beta) / \beta}(t-m) .
\end{aligned}
$$

For the case $\beta=\gamma$, we see that $y^{(\gamma-\beta) / \beta}(t)=1$ while for the case $\beta>\gamma$ and since $(a(t)(\Delta y(t)))^{\gamma}$ is decreasing, there exists a constant $c>0$ such that

$$
(a(t)(\Delta y(t)))^{\gamma} \leqslant c \text { for } t \geqslant t_{2} .
$$

Summing this inequality from $t_{2}$ to $t-1$, we have

$$
y(t) \leqslant y\left(t_{2}\right)+c^{1 / \gamma} A\left(t, t_{2}\right),
$$

and thus,

$$
y^{(\gamma-\beta) / \beta}(t) \geqslant c^{(\gamma-\beta) /(\beta \gamma)} A^{(\gamma-\beta) / \beta}\left(t, t_{2}\right):=c^{*} A^{(\gamma-\beta) / \beta}\left(t, t_{2}\right),
$$


where $c^{*}=c^{(\gamma-\beta) /(\beta \gamma)}$. Using those two cases and the definition of $g(t)$, we get

$$
\begin{aligned}
\Delta w(t) \leqslant & -\rho(t) q(t)+\left(\frac{\Delta \rho(t)}{\rho(t+1)}\right) w(t+1) \\
& -\frac{\beta \rho(t)}{a^{1 / \gamma}(t-m) \rho^{1+1 / \gamma}(t+1)} g(t) w^{(1+\gamma) / \gamma}(t+1) .
\end{aligned}
$$

Setting

and using

$$
B:=\left(\frac{\Delta \rho(t)}{\rho(t+1)}\right) \text { and } C:=\frac{\beta \rho(t)}{a^{1 / \gamma}(t-m) \rho^{1+1 / \gamma}(t+1)},
$$

$$
B u-C u^{(1+\gamma) / \gamma} \leqslant \frac{\gamma^{\gamma}}{(1+\gamma)^{\gamma+1}}\left(\frac{B^{\gamma+1}}{C^{\gamma}}\right),
$$

(see [7]), we have

$$
\Delta w(t) \leqslant-\rho(t) q(t)+\frac{\gamma^{\gamma}}{(1+\gamma)^{\gamma+1}} \frac{a(t-m)}{(\beta g(t))^{\gamma}}\left(\frac{(\Delta \rho(t))^{\gamma+1}}{\rho^{\gamma}(t)}\right) .
$$

Summing this inequality from $t_{2}$ to $t-1$ we get

$$
w(t) \leqslant w\left(t_{2}\right)-\sum_{s=t_{2}}^{t-1}\left[\rho(s) q(s)-\frac{\gamma^{\gamma}}{(1+\gamma)^{\gamma+1}} \frac{a(s-m)}{(\beta g(s))^{\gamma}}\left(\frac{(\Delta \rho(s))^{\gamma+1}}{\rho^{\gamma}(s)}\right)\right] .
$$

Taking into account the equation (12), we find

$$
w\left(t_{2}\right) \geqslant \rho(t) Q(t)+\sum_{s=t_{2}}^{t-1}\left[\rho(s) q(s)-\frac{\gamma^{\gamma}}{(1+\gamma)^{\gamma+1}} \frac{a(s-m)}{(\beta g(s))^{\gamma}}\left(\frac{(\Delta \rho(s))^{\gamma+1}}{\rho^{\gamma}(s)}\right)\right] .
$$

Taking the limsup of both sides in the above inequality as $t \rightarrow \infty$, we obtain a contradiction to the equation(3).

Consider now case (II). If we put $z(t)=-y(t)>0$ for $t \geqslant t_{2}$, then

$$
z(t)=-y(t)=p(t) x(t-k)-x(t) \leqslant p(t) x(t-k),
$$

or

$$
x(t-k) \geqslant z(t) \text { or } z(t)=x(t+k) .
$$

Using this inequality in equation (1), we have

$$
\Delta\left(a(t)(\Delta z(t))^{\gamma}\right) \geqslant q(t) z^{\beta}(t-m+k+1):=q(t) z^{\beta}(h(t)) .
$$

Clearly, we have $\Delta z(t)<0$. Now, for $t_{2} \leqslant u \leqslant v$, we may write $z(u)-z(v)=-\sum_{s=u}^{v}\left(a^{-1 / \gamma}(s)\left(a(s)(\Delta z(s))^{\gamma}\right)^{1 / \gamma}\right) \geqslant A(v, u)\left(-\left(a(v)(\Delta z(v))^{\gamma}\right)^{1 / \gamma}\right)$, for $t \geqslant s \geqslant t_{2}$, setting $u=h(s)$ and $v=h(t)$ in the above inequality we get

$$
z(h(s)) \geqslant A(h(t), h(s))\left(-\left(a(h(t))(\Delta z(h(t)))^{\gamma}\right)^{1 / \gamma}\right) .
$$

Summing inequality (18) from $u=h(t) \geqslant t_{2}$ to $t-1$, we find 


$$
\begin{aligned}
Z(t) & :=-a(h(t))(\Delta z(h(t)))^{\gamma} \\
& \geqslant\left(-a(h(t))(\Delta z(h(t)))^{\gamma}\right)^{\frac{\beta}{\gamma}} \sum_{s=h(t)}^{t-1} A^{\beta}(h(t), h(s)) q(s) \\
& =Z^{\beta / \gamma}(t) \sum_{s=h(t)}^{t-1} A^{\beta}(h(t), h(s)) q(s),
\end{aligned}
$$

and hence

$$
Z^{1-\beta / \gamma} \geqslant \sum_{s=h(t)}^{t-1} A^{\beta}(h(t), h(s)) q(s) .
$$

Taking limsup of both sides of this inequality as $t \rightarrow \infty$, we arrive at a contradiction to equation (5) when $\beta=\gamma$ and equation (6) when $\beta>\gamma$. This completes the proof.

We note that Theorem 2.1 holds when $Q(t)<\infty$ and the additional term $\rho(t) Q(t)$ in equation (3) may improves some of the well-known existing results appeared in the literature. In the case when $Q(t)$ does not exists as $t \rightarrow \infty$, we see that equation (3) can be replaced by

$$
\limsup _{t \rightarrow \infty} \sum_{s=t_{2}}^{t-1}\left[\rho(s) q(s)-\frac{\gamma^{\gamma}}{(1+\gamma)^{\gamma+1}} \frac{a(t-m+1)}{(\beta g(s))^{\gamma}}\left(\frac{(\Delta \rho(s))^{\gamma+1}}{\rho^{\gamma}(s)}\right)\right]=\infty,
$$

and the conclusion of Theorem 2.1 holds.

For the non-neutral equations, that is, equation (1) when $p(t)=0$ and $q(t)$ is either non-negative or non-positive for all large $t$, equation (1) is reduced to the equation

$$
\Delta\left(a(t)(\Delta x(t))^{\gamma}\right)+\delta q(t) x^{\beta}(t+1-m)=0,
$$

where $\delta= \pm 1$. From Theorem 2.1, we extract the following immediate results.

Corollary 2.2. Let conditions (i)-(iii) and equation (2) hold. If there exists a positive function $\rho(t)$ and $\Delta \rho(t) \geqslant 0$ such that equation (3) holds, then equation $(1,+1)$ is oscillatory.

Proof. The proof is contained in the proof of Theorem 2.1-Case (I) and hence is omitted.

We note that Corollary 2.2 is related to some of the results in $[4,5,6,12,13$, $14,15,16,17]$ and the references cited therein.

Corollary 2.3. Let conditions (i)-(iv) and equation (2) hold. If equation (5) or (6) holds, then every bounded solution of equation $(1,-1)$ is oscillatory.

Proof. The proof is contained in the proof of Theorem 2.1-Case (II) and hence is omitted.

The following examples are illustrative. 
Example 2.4. Consider the neutral equation

$$
\Delta^{2}\left(x(t)-\frac{1}{2} x(t-3)\right)+8 x(t-7)=0 .
$$

Here, $k=3$ and $m=8$ and so, $h(t)=t-3$. All conditions of Theorem 2.1 with equation (3) be replaced by equation (19) are satisfied and hence equation (20) is oscillatory.

Next, we present the following interesting results.

Theorem 2.5. Let the hypotheses of Theorem 2.1 hold with $\Delta \rho \leqslant 0$ for $t \geqslant t_{0}$ and equation (3) be replaced by

$$
\limsup _{t \rightarrow \infty}\left[\rho(t) Q(t)+\sum_{s=t_{0}}^{t-1} \rho(s) q(s)\right]=\infty .
$$

Then equation (1) is oscillatory.

Proof. Let $x(t)$ be a non-oscillatory solution of equation (1), say $x(t)>0, x(t-$ $k)>0, x(t-m+1)>0$ for $t \geqslant t_{1}$. Proceeding as in the proof of Theorem 2.1, we conclude that $\Delta y(t)>0$ for $t \geqslant t_{2}$ and we have two cases to consider: (I) $y(t)>0$ or $y(t)<0$ for $t \geqslant t_{2}$.

Case (I). Suppose that $y(t)>0$. As in the proof of Theorem 2.1, we obtain (16). Thus,

$$
\Delta w(t) \leqslant-\rho(t) q(t) .
$$

Summing this inequality and using equation (10) we arrived at the desired contradiction.

Example 2.6. Consider the neutral equation

$$
\Delta^{2}\left(x(t)-\frac{1}{2} x(t-1)\right)+x(t-1)=0 .
$$

Here, $k=1$ and $m=1$ and so, $\rho(t)=t$. All conditions of Theorem 2.1 with equation (3) be replaced by equation (21) are satisfied and hence equation (22) is oscillatory.

In the following theorem we employ different approaches to replace equation (3) in Theorem 2.1.

Theorem 2.7. Let the hypotheses of Theorem 2.1 hold with $\gamma \leqslant 1$, and equation (3) be replaced by

$$
\limsup _{t \rightarrow \infty}\left[\rho(t) Q(t)+\sum_{s=t_{0}}^{t-1} \rho(s) q(s)-\frac{a^{1 / \gamma}(s-m+1)(\Delta \rho(s))^{2}}{4 \beta g(s) \rho(s) Q^{1 / \gamma-1}(s+1)}\right]=\infty
$$

Then equation (1) is oscillatory. 
Proof. Let $x(t)$ be a non-oscillatory solution of equation (1), say $x(t)>0, x(t-$ $k)>0, x(t-m+1)>0$ for $t \geqslant t_{1}$. Proceeding as in the proof of Theorem 2.1, we conclude that $\Delta y(t)$ for $t \geqslant t_{2}$ and $y(t)$ satisfies either (I) or (II) for $t \geqslant t_{2}$. If (I) holds, then as in the proof of Theorem 2.1, we obtain (17) and using (12) we get

$$
\begin{aligned}
& \Delta w(t) \leqslant-\rho(t) q(t)+\left(\frac{\Delta \rho(t)}{\rho(t+1)}\right) w(t+1) \\
& -\frac{\beta \rho(t)}{a^{1 / \gamma}(t-m+1) \rho^{1+1 / \gamma}(t+1)} g(t) w^{1+1 / \gamma}(t+1) \\
& \leqslant-\rho(t) q(t)+\left(\frac{\Delta \rho(t)}{\rho(t+1)}\right) w(t+1) \\
& -\frac{\beta \rho(t)}{a^{1 / \gamma}(t-m+1) \rho^{2}(t+1)} g(t) Q^{1 / \gamma-1}(t+1) w^{2}(t+1)
\end{aligned}
$$

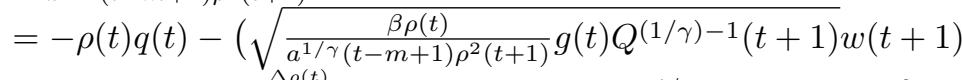

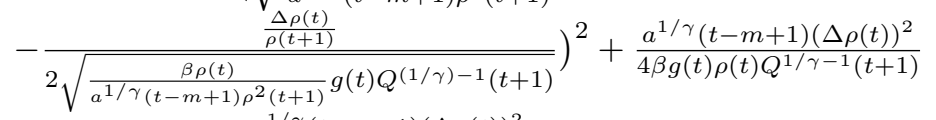

$$
\begin{aligned}
& \leqslant-\rho(t) q(t)+\frac{a^{1 / \gamma}(t-m+1)(\Delta \rho(t))^{2}}{4 \beta g(t) \rho(t) Q^{(1 / \gamma)-1}(t+1)} .
\end{aligned}
$$

The rest of the proof is similar to that of Theorem 2.1 and hence is omitted.

Example 2.8. Consider the neutral equation

$$
\Delta^{2}\left(x(t)-\frac{1}{3} x(t-2)\right)+x(t-3)=0 .
$$

Here, $k=2$ and $m=4$ and so, $\gamma=1, \rho(t)=t$. All conditions of Theorem 2.1 with equation (3) be replaced by equation (23) are satisfied and hence equation (24) is oscillatory.

Next, we present the following new and easily verifiable oscillation criteria for equation (1).

Theorem 2.9. Let conditions (i)-(iv) and equation (2) hold. Assume that equation (5) and

$$
\limsup _{t \rightarrow \infty} A^{\beta}\left(t-m+1, t_{0}\right) Q(t)>1
$$

hold when $\beta=\gamma$, and equation (6) and

$$
\limsup _{t \rightarrow \infty} A^{\beta}\left(t-m+1, t_{0}\right) Q(t)>0
$$

hold when $\beta<\gamma$, then equation (1) is oscillatory.

Proof. Let $x(t)$ be a non-oscillatory solution of equation (1), say $x(t)>0, x(t-$ $k)>0, x(t-m+1)>0$ for $t \geqslant t_{1}$ for some $t_{1} \geqslant t_{0}$. Proceeding as in the proof of Theorem 2.1, we conclude that $\Delta y(t)>0$ for $t \geqslant t_{2}$ and $y(t)$ satisfies either (I) or (II) for $t \geqslant t_{2}$. If (I) holds, then as in the proof of Theorem 2.1, we obtain 
(9) and (10). Using the facts that $\sigma(t) \leqslant t$ is decreasing, we find

$$
\begin{aligned}
& w(t):=a(t)(\Delta y(t))^{\gamma} \geqslant Q(t) \mu^{\beta}(\tau(t))(\Delta y(t-m+1))^{\beta} \\
& =Q(t) \mu^{\beta}(t-m+1)\left(a^{-\beta / \gamma}(t-m+1)\right)\left(a(t-m+1)(\Delta y(t-m+1))^{\gamma}\right)^{\beta / \gamma} \\
& \geqslant Q(t) \mu^{\beta}(t-m+1)\left(a^{-\beta / \gamma}(t-m+1)\right)\left(a(t)(\Delta y(t))^{\gamma}\right)^{\beta / \gamma} \\
& =Q(t) \mu^{\beta}(t-m+1)\left(a^{-\beta / \gamma}(t-m+1)\right) w^{\beta / \gamma}(t),
\end{aligned}
$$

or

$$
\begin{aligned}
w^{1-\beta / \gamma}(t) & \geqslant Q(t) \mu^{\beta}(t-m+1)\left(a^{-\beta / \gamma}(\tau(t))\right. \\
& =Q(t)\left(\sum_{s=t_{2}}^{t-m+1} a^{-1 / \gamma}(s)\right)^{\beta}=A^{\beta}\left(t-m+1, t_{2}\right) Q(t) .
\end{aligned}
$$

Taking limsup of both sides of this inequality as $t \rightarrow \infty$, we arrive at a contradiction to equation (25) when $\beta=\gamma$ and equation (26) when $\beta<\gamma$. The proof of case (II) is similar to that of Theorem 2.1 and hence is omitted.

Example 2.10. Consider the neutral equation

$$
\Delta\left(\Delta\left(x(t)-\frac{1}{3} x(t-2)\right)\right)^{2}+x(t-3)=0 .
$$

Here, $k=2$ and $m=4$ and so, $\gamma=2, \beta=1$. Equation (26) of Theorem 2.5 are satisfied and hence equation (27) is oscillatory.

For equation (1) with advanced argument, we present the following result.

Theorem 2.11. Let $\tau(t) \geqslant t$, conditions ( $i)$-(iii) and equation (2) hold. Assume that the conditions

$$
\begin{gathered}
\limsup _{t \rightarrow \infty} A\left(t, t_{0}\right) Q^{1 / \gamma}(t)>1 \\
\limsup _{t \rightarrow \infty} \sum_{u=h(t)}^{t-1}\left(\frac{1}{a(u)} \sum_{s=u}^{t} q(s)\right)^{1 / \gamma}>1
\end{gathered}
$$

hold when $\gamma=\beta$ and the conditions

$$
\begin{aligned}
& \limsup _{t \rightarrow \infty} A\left(t, t_{0}\right) Q^{1 / \gamma}(t)=\infty \\
& \limsup _{t \rightarrow \infty} \sum_{u=h(t)}^{t-1}\left(\frac{1}{a(u)} \sum_{s=u}^{t} q(s)\right)^{1 / \gamma}>0
\end{aligned}
$$

hold when $\beta<\gamma$, then equation (1) is oscillatory. 
Proof. Let $x(t)$ be a non-oscillatory solution of equation (1), say $x(t)>0, x(t-$ $k)>0, x(t-m+1)>0$ for $t \geqslant t_{1}$ for some $t_{1} \geqslant t_{0}$. Proceeding as in the proof of Theorem 2.1 and consider the two cases (I) and (II). First, suppose case (I) holds. From equation (10), we have

$$
(\Delta y(t))^{\gamma} \geqslant\left(\frac{Q(t)}{a(t)}\right) y^{\beta}(t-m+1),
$$

or

$$
\Delta y(t) \geqslant\left(\frac{Q(t)}{a(t)}\right)^{1 / \gamma} y^{\beta / \gamma}(t-m+1) .
$$

Using above inequality in (9), we get

$$
\begin{aligned}
y(t) & \geqslant \mu(t) \Delta y(t) \\
& \geqslant \mu(t)\left(\frac{1}{a(t)} \sum_{s=t}^{\infty} q(s)\right)^{1 / \gamma} y^{\beta / \gamma}(t-m+1) \\
& \geqslant A\left(t, t_{2}\right) Q^{1 / \gamma}(t) y^{\beta / \gamma}(t),
\end{aligned}
$$

or

$$
y^{1-\beta / \gamma}(t) \geqslant A\left(t, t_{2}\right) Q^{1 / \gamma}(t)
$$

Taking limsup of both sides of this inequality as $t \rightarrow \infty$, we arrive at a contradiction to equation (28) when $\beta=\gamma$ and equation (30) when $\beta<\gamma$. If (II) holds, then as in the proof of Theorem 2.1-Case (II), we obtain equation (18). Summing this inequality from $u$ to $t-1$,

$$
(a(t)(\Delta z(t)))^{\gamma}-(a(u)(\Delta z(u)))^{\gamma} \geqslant \sum_{s=u}^{t} q(s) z^{\beta}(h(s))
$$

or

$$
-\Delta z(u) \geqslant\left(\frac{1}{a(u)} \sum_{s=u}^{t} q(s) z^{\beta}(h(s))\right)^{1 / \gamma} \geqslant\left(\frac{1}{a(u)} \sum_{s=u}^{t} q(s)\right)^{1 / \gamma} z^{\beta / \gamma}(h(t)) .
$$

Summing this inequality from $h(t) \geqslant t_{2}$ to $t-1$, we arrive at a contradiction to equation (29) when $\beta=\gamma$ or equation (31) when $\beta<\gamma$.

Example 2.12. Consider the neutral equation

$$
\Delta\left(\Delta\left(x(t)-\frac{1}{3} x(t-2)\right)\right)^{2}+2 x(t-3)=0 \text {. }
$$

Here, $k=2$ and $m=4$ and so, $\gamma=2, \beta=1$. Condition (30) and (31) of Theorem 2.5 are satisfied and hence equation (32) is oscillatory.

We may note that corollaries similar to Corollaries 2.2 and 2.3 can be also drawn from Theorems 2.5 and 2.7. The details are left to the reader. 


\section{Conclusion}

We present seven sufficient conditions which ensure that all solutions of (1) are oscillatory. The corresponding examples are given to illustrate the significance of the results. From this, the oscillation criteria for the $n$ order equation are similar.

\section{Competing Interests}

The author(s) do not have any competing interests in the manuscript.

\section{Acknowledgments}

The second author is supported by Shandong Provincial Natural Science Foundation (ZR2016AM17).

\section{REFERENCES}

1. Agarwal, R. P., Grace, S. R., \& OŔegan, D. (2002). Oscillation theory for second order linear, half-linear, superlinear and sublinear dynamic equations. Springer Science \& Business Media.

2. Agarwal, R. P. (2000). Difference equations and inequalities: theory, methods, and applications. CRC Press.

3. Agarwal, R. P., Bohner, M., Grace, S. R., \& ORegan, D. (2005). Discrete Oscillation Theory, Hindawi Publ. Corp., New York.

4. Agarwal, R. P. \& Grace, S. R. (2001). Oscillation of certain third order difference equations. Computers \& Mathematics with Applications, 42(3-5), 379-384. https://doi.org/10.1016/S0898-1221(01)00162-6

5. Agarwal, R. P., Grace, S. R., \& ORegan, D. (2000). Oscillation Theory for Difference and Functional Differential Equations. Springer Netherlands.

6. Agarwal, R. P., Bohner, M., Li, T., \& Zhang, C. (2014). Oscillation of second-order differential equations with a sublinear neutral term. Carpathian Journal of Mathematics, 30(1), 1-6. http://dx.doi.org/10.15826/umj.2017.2.013

7. El-Morshedy, H. A. (2006). Oscillation and nonoscillation criteria for half-linear second order difference equations. Dynamic Systems and Applications, 15(3), 429-450.

8. El-Morshedy, H. A. (2009). New oscillation criteria for second order linear difference equations with positive and negative coefficients. Computers \& Mathematics with Applications, 58(10), 1988-1997. https://doi.org/10.1016/j.camwa.2009.07.078

9. Grace, S. R., Agarwal, R. P., Bohner, M. \& OŔegan, D. (2009). Oscillation of second order strongly superlinear and strongly sublinear dynamic equations. Communications in Nonlinear Science and Numerical Simulation, 14(8), 3463-3471. https://doi.org/10.1016/j.cnsns.2009.01.003

10. Grace, S. R., Agarwal,R. P., Kaymakalan, B. \& Sae-jie, W. (2010). Oscillation theorems for second order nonlinear dynamic equations. Journal of Applied Mathematics and Computing, 32(1), 205-218. https://doi.org/10.1007/s12190-009-0244-7

11. Grace, S. R., Bohner, M., \& Agarwal, R. P. (2009). On the oscillation of second-order half-linear dynamic equations. Journal of Difference Equations and Applications, 15(5), 451-460. https://doi.org/10.1080/10236190802125371

12. Grace, S. R., \& El-Morshedy, H. A. (2000). Oscillation criteria of comparison type for second order difference equations. Journal of Applied Analysis, 6(1), 87-102. https://doi.org/10.1515/JAA.2000.87 
13. El-Morshedy, H. A., \& Grace, S. R. (2005). Comparison theorems for second order nonlinear difference equations. Journal of mathematical analysis and applications, 306(1), 106-121. https://doi.org/10.1016/j.jmaa.2004.12.024

14. Liu, X. (2006). Oscillation of solutions of neutral difference equations with a nonlinear term. Comp. Math. Appl, 52(3-4), 439-448. https://doi.org/10.1016/j.camwa.2006.02.009

15. Tang, X. H. (2001). Oscillation for nonlinear delay difference equations. Tamkang Journal of Mathematics, 32(4), 275-280.

16. Thandapani, E., Liu, Z. S., Arul, R., \& Palanisamy S. Raja. (2004). Oscillation and asymptotic behavior of second order difference equations with nonlinear neutral terms. Applied Mathematics E - Notes, 4, 59-67. https://doi.org/10.4236/jamp.2017.56104

17. Thandapani, E., Pandian, S., \& Balasubramanian, R. K. (2004). Oscillation of solutions of non- linear neutral difference equations with nonlinear neutral term. Far East Journal of Applied Mathematics, 15, 47-62. http://dx.doi.org/10.17654/MS104010091

18. Thandapani, E., \& Mahalingam, K. (2003). Necessary and sufficient conditions for oscillation of second order neutral difference equations. Tamkang Journal of Mathematics, $34(2), 137-145$.

19. Thandapani, E., Mahalingam, K., \& Graef, J. R. (2003). Oscillatory and asymptotic behavior of second order neutral type difference equations. International Journal of Pure \& Applied Mathematics, 6(2), 217-230.

20. Yang, J., Guan, X., \& Liu, W. (1997). Oscillation and asymptotic behavior of second order neutral difference equation. Ann. Diff. Equ., 13, 94-106.

21. Yildiz, M. K., \& Ogunmez, H. (2014). Oscillation results of higher order nonlinear neutral delay difference equations with a nonlinear neutral term. Hacettepe University Bulletin of Natural Sciences \& Engineering, 43(5), 809-814.

22. Zhang, Z., Chen, J. \& Zhang, C. (2001). Oscillation of solutions for second order nonlinear differ- ence equations with nonlinear neutral term. Computers \& Mathematics with Applications, 41(12), 1487-1494. https://doi.org/10.1016/S0898-1221(01)00113-4

Said R. Grace

Department of Engineering Mathematics, Faculty of Engineering Cairo University, Orman, Giza 12221, Egypt.

e-mail: saidgrace@yahoo.com

Shurong Sun

School of Mathematical Science, University of Jinan, Jinan, Shandong 250022, P R China. e-mail: sshrong@163.com

Limei Feng

School of Mathematical Science, University of Jinan, Jinan, Shandong 250022, P R China. e-mail: fenglimei1004@163.com

Ying Sui

School of Mathematical Science, University of Jinan, Jinan, Shandong 250022, P R China.

e-mail: suiying4320@163.com 